\title{
Model to Describe Fast Shutoff of CoVID-19 Pandemic Spread
}

\author{
Genghmun Eng \\ PhD Physics 1978, University of Illinois at Urbana-Champaign \\ geng001@socal.rr.com
}

August 6, 2020

\begin{abstract}
Early CoVID-19 growth obeys: $N\{\widehat{t}\}=N_{I} \exp \left[+K_{o} \widehat{t}\right]$, with $K_{o}=$ $\left[(\ln 2) /\left(t_{d b l}\right)\right]$, where $t_{d b l}$ is the pandemic growth doubling time. Given $N\{\widehat{t}\}$, the daily number of new CoVID-19 cases is $\rho\{\widehat{t}\}=d N\{\widehat{t}\} / d \widehat{t}$. Implementing society-wide Social Distancing increases the $t_{d b l}$ doubling time, and a linear function of time for $t_{d b l}$ was used in our Initial Model:$$
N_{o}[t]=1 \exp \left[+K_{A} t /\left(1+\gamma_{o} t\right)\right] \equiv e^{+G_{o}} \exp \left(-Z_{o}[t]\right),
$$

to describe these changes, with $G_{o} \equiv\left[K_{A} / \gamma_{o}\right]$. However, this equation could not easily model some quickly decreasing $\rho[t]$ cases, indicating that a second Social Distancing process was involved. This second process is most evident in the initial CoVID-19 data from China, South Korea, and Italy. The Italy data is analyzed here in detail as representative of this second process. Modifying $Z_{o}[t]$ to allow exponential cutoffs:

$Z_{E}[t] \equiv+\left[G_{o} /\left(1+\gamma_{o} t\right)\right]\left[\exp \left(-\delta_{o} t-q_{o} t^{2}\right)\right]=Z_{o}[t] \exp \left(-\delta_{o} t-q_{o} t^{2}\right)$, provides a new Enhanced Initial Model (EIM), which significantly improves datafits, where $N_{E}[t]=e^{+G_{o}} \exp \left(-Z_{E}[t]\right)$. Since large variations are present in $\rho_{\text {data }}[t]$, these models were generalized into an orthogonal function series, to provide additional data fitting parameters:

$$
N(Z)=\sum_{m=0}^{m=M_{F}} g_{m} L_{m}(Z) \exp [-Z] .
$$

Its first term can give $N_{o}[t]$ or $N_{E}[t]$, for $Z[t] \rightarrow Z_{o}[t]$ or $Z[t] \rightarrow Z_{E}[t]$. The $L_{m}(Z)$ are Laguerre Polynomials, with $L_{0}(Z)=1$, and $\left\{g_{m} ; m=\right.$ $\left.0, M_{F}\right\}$ are constants derived from each dataset. When $\rho[t]=d N[t] / d t$ gradually decreases, using $Z_{o}[t]$ provided good datafits at small $M_{F}$ values, but was inadequate if $\rho[t]$ decreased faster. For those cases, $Z_{E}[t]$ was used in the above $N(Z)$ series to give the most general Enhanced Orthogonal Function [EOF] model developed here. Even with $M_{F}=0, q_{o}=0$, this $E O F$ model fit the Italy CoVID-19 data for $\rho[t] \equiv d N[t] / d t$ fairly well.

When the $\rho[t]$ post-peak behavior is not Gaussian, then $Z_{E}[t]$ with $\delta_{o} \neq 0, q_{o}=0$; which we call $Z_{A}[t]$, is also likely to be a sufficient extension of the $Z_{o}[t]$ model. The EOF model also can model a gradually decreasing $\rho[t]$ tail using small $\left\{\delta_{o}, q_{o}\right\}$ values [with 6 Figures].
\end{abstract}




\section{Introduction}

Let $N\{\hat{t}\}$ be the total number of CoVID-19 cases in any given locality, with $\rho\{\widehat{t}\}$ being the predicted number of daily new CoVID-19 cases, so that:

$$
\begin{aligned}
& N\{\widehat{t}\}=\int_{t^{\prime}=0}^{t^{\prime}=\widehat{t}} \rho\left\{t^{\prime}\right\} d t^{\prime}, \\
& \rho\{\widehat{t}\}=d N\{\widehat{t}\} / d \widehat{t} .
\end{aligned}
$$

Early CoVID-19 growth often obeys $N\{\widehat{t}\} \approx N_{I} \exp \left[+K_{o} \widehat{t}\right]$, with $K_{o}=$ $\left[(\ln 2) / t_{d b l}\right]$, where $t_{d b l}$ is the pandemic doubling time. The start of societywide Social Distancing at $\widehat{t}=0$ can gradually lengthen $t_{d b l}$ for $\widehat{t}>0$. The $\widehat{t}<0$ exponential growth phase is not applicable for estimating Social Distancing effects. For $\widehat{t}>0$, an Initial Model for CoVID-19 pandemic shutoff was first developed ${ }^{1}$ using a linear function of time to describe the $t_{d b l}$ changes:

$$
N\{\widehat{t}\}=N_{I} \exp \left[+K_{o} \widehat{t} /\left(1+\alpha_{S} \widehat{t}\right)\right] .
$$

Given measured $N_{\text {data }}\{\widehat{t}\}$, the data end-points $\left\{N_{I}, N_{F}\right\}$ help to set $\left\{K_{o}, \alpha_{S}\right\}$. An Orthogonal Function Model [OFM] was developed next ${ }^{2}$, with Eq. [1.2] as the first term of the orthogonal function series. Each new OFM term provides another fitting parameter, to progressively better match $N_{\text {data }}\{\widehat{t}\}$ and $\rho_{\text {data }}\{\widehat{t}\}=(d / d \widehat{t}) N_{\text {data }}\{\widehat{t}\}$.

The OFM improves on the Initial Model, and it works best with gradually decreasing $\rho\{\hat{t}\}$ ["Slow Shutoff"]. In contrast, when $\rho\{\widehat{t}\}$ decreased quickly ["Fast Shutoff"], the Initial Model was not a good datafit, and a few-term OFM series only gave small improvements. This result indicates there is an inherent limit to what the gradually changing $t_{d b l}$ doubling time of Eq. [1.2] can model.

For these cases, typified by CoVID-19 pandemic evolution in Italy, data often showed a stage where $\rho_{\text {data }}\{\widehat{t}\} \sim\left[\exp \left(-\delta_{o} \widehat{t}\right)\right]$ or $\rho_{\text {data }}\{\widehat{t}\} \sim\left[\exp \left(-q_{o} \widehat{t}^{2}\right)\right]$, which likely represents a second process, independent of the gradually changing $t_{d b l}$ doubling time. An Enhanced Initial Model (EIM) is developed here to include this second process. The prior $O F M$ methods can then be applied, giving an Enhanced Orthogonal Function (EOF) model for this more general case.

\subsection{Review of Prior Models}

The Initial Model of Eq. [1.2] is still needed as the first part of the OFM. The Initial Model starts with measured data end-points $\left\{N_{I}, N_{F}\right\}$, where $\widehat{t}=$ $\left(t_{F}-t_{I}\right)$ is the largest data time interval so that $N\left\{\widehat{t}=\left(t_{F}-t_{I}\right)\right\}=N_{F}$. Usually $\alpha_{S}$ in Eq. [1.2] was chosen first, and $K_{o}$ or $t_{d b l}$ adjusted to match the $N_{F}$ data end-point, using an Excel ${ }^{T M}$ _Goal-Seek or its equivalent. The final $\left\{K_{o}, \alpha_{S}\right\}$ values were the pair with the minimum root-mean-square (rms) error between the given data and the Eq. [1.2] model.

The above $\left\{K_{o}, \alpha_{S}\right\}$ also provides a $t=0$ estimate for the pandemic start, and gives $\left\{K_{A}, \gamma_{o}\right\}$ as new data fitting parameters:

$$
\begin{aligned}
& N_{o}[t]=\mathbf{1} \exp \left[+K_{A} t /\left(1+\gamma_{o} t\right)\right] \equiv \exp \left[+G_{o}\right] \exp \left(-Z_{o}[t]\right) \\
& G_{o} \equiv\left(K_{A} / \gamma_{o}\right) \\
& Z_{o}[t]=+\left[G_{o} /\left(1+\gamma_{o} t\right)\right]
\end{aligned}
$$


$\rho_{o}[t] \equiv d N_{o}[t] / d t$

Using $\left(t_{F}-t_{I}\right)$, with $N_{o}\left[t=t_{I}\right]=N_{I}$ and $N_{o}\left[t=t_{F}\right]=N_{F}$, sets:

$t_{I}=\ln \left(N_{I}\right) /\left[K_{o}+\alpha_{S} \ln \left(N_{I}\right)\right]$.

$t_{F}=\ln \left(N_{I}\right) /\left[K_{o}+\alpha_{S} \ln \left(N_{I}\right)\right]+\left(t_{F}-t_{I}\right)$,

which determines $\left\{K_{A}, \gamma_{o}\right\}$ in $Z_{o}[t]$ for Eq. [1.3c]:

$$
\begin{aligned}
& \gamma_{o}=\left\{\left[\ln \left(N_{I}\right) / t_{I}\right]-\left[\ln \left(N_{F}\right) / t_{F}\right]\right\} /\left[\ln \left(N_{F}\right)-\ln \left(N_{I}\right)\right], \\
& K_{A}=\left[\left(1 / t_{I}\right)-\left(1 / t_{F}\right)\right] /\left\{\left[1 / \ln \left(N_{I}\right)\right]-\left[1 / \ln \left(N_{F}\right)\right]\right\} .
\end{aligned}
$$

The Eq. [1.4a] value for $t_{I}$ is what determines the new $t=0$ point, as an extrapolation for when $N_{o}[t=0]=\mathbf{1}$. In addition:

$N_{o}\left[t \rightarrow \infty, Z_{o} \rightarrow 0\right] \approx \mathbf{1} \exp \left[+K_{A} / \gamma_{o}\right]=\exp \left[+G_{o}\right] \equiv N_{\max }^{o}$,

$\rho_{o}[t]=d N_{o}[t] / d t \approx N_{o}[t]\left[G_{o} \gamma_{o} /\left(1+\gamma_{o} t\right)^{2}\right] \rightarrow N_{\max }^{o}\left[G_{o} /\left(\gamma_{o} t^{2}\right)\right], \quad[1.6 \mathrm{~b}]$

provides an estimate for the total number of cases $\left(N_{\max }^{o}\right)$ at the pandemic end, and determines a function for the $\rho_{o}[t]$ long-time tail. The $\{0<t<$ $\left.t_{I}\right\}$ period prior to the start of Social Distancing, extrapolates what pandemic progress would have looked like, if Social Distancing had begun at $t=0$.

An Orthogonal Function Model [OFM] was then developed ${ }^{2}$ to better model the different observed $\rho_{\text {data }}[t]$ shapes, as an improvement of the Initial Model:

$$
\begin{aligned}
N(Z) & =\sum_{\substack{m=0 \\
m=M_{F}}} g_{m} L_{m}(Z) \exp [-Z], \\
R(Z) & =\sum_{\substack{m=0 \\
m=M_{F}}} c_{m} L_{m}(Z) \exp [-Z], \\
N(Z) & \equiv \int_{Z^{\prime}=+\infty} R\left(Z^{\prime}\right) d Z^{\prime}, \\
c_{M_{F}-k} & =\sum_{m=0}^{m=k} g_{m},
\end{aligned}
$$

with $L_{m}(Z)$ being the Laguerre Polynomials, and $L_{m}(Z=0)=L_{0}(Z) \equiv 1$. Using $Z=Z_{o}[t]$ from Eq. [1.3c] gives $N(Z) \rightarrow N\left(Z_{o}\right)$ and $R(Z) \rightarrow R\left(Z_{o}\right)$.

The $\left\{g_{m} ; m=\left(0, M_{F}\right)\right\}$ constants in Eq. [1.7a] can be arranged in a $\vec{g}$-vector form, with comparable constants for $R(Z)$ from Eq. [1.7b] arranged in a $\vec{C}$-vector form. For $M_{F}=2$, it allows Eq. [1.7d] to be written as:

$$
\vec{C}=\left(\begin{array}{l}
c_{0} \\
c_{1} \\
c_{2}
\end{array}\right)=\left(\begin{array}{lll}
1 & 1 & 1 \\
0 & 1 & 1 \\
0 & 0 & 1
\end{array}\right) \vec{g}=\left(\begin{array}{ccc}
1 & 1 & 1 \\
0 & 1 & 1 \\
0 & 0 & 1
\end{array}\right)\left(\begin{array}{l}
g_{0} \\
g_{1} \\
g_{2}
\end{array}\right) .
$$

Once these $c_{m}$ values are determined for $R(Z)$ in Eqs. [1.7b]-[1.7c], an $O F M$ feature is that $c_{0}$, by itself, becomes the $O F M$ best estimate for the total number of CoVID-19 cases at the pandemic end.

For large enough $M_{F}$ values, and a monotonic $Z$-function, this $O F M$ can provide successively better approximations to almost any given set of $N_{\text {data }}[Z]$, with $Z[t] \rightarrow Z_{o}[t]$ of Eq. [1.3c] being a specific case.

The OFM implicitly uses a Linear $Y$-axis, so its results differ from the Initial Model datafit on a Logarithmic Y-axis. As an example, compare the Initial Model result of $N_{o}\left(Z_{o}\right)=G_{0} \exp \left[-Z_{o}\right]$ with the Eq. [1.7a] OFM result of $N\left(Z_{o}\right)=g_{0} \exp \left[-Z_{o}\right]$ for $M_{F}=0$. In the Initial Model, $G_{o}$ is fixed so that $N_{o}\left(Z_{o}\right)$ exactly matches $\left\{N_{I}, N_{F}\right\}$ at the $\left\{t_{I}, t_{F}\right\}$ boundaries. In the OFM, 
$g_{0}=G_{o}$ is no longer required, so that the $O F M N\left(Z_{o}\right)$ best datafit is not constrained to exactly match $\left\{N_{I}, N_{F}\right\}$ at $\left\{t_{I}, t_{F}\right\}$.

The above $R(Z)$ and $Z[t]$ gives $N[t]$ and $\rho[t]$ as an explicit functions of time:

$$
N[t] \equiv \int_{\widehat{z}=Z[t], t^{\prime}=t}^{\widehat{z}=+\infty, t^{\prime}=\left(t_{\min }\right)} R\left(Z\left[t^{\prime}\right]\right) \frac{d Z}{d t^{\prime}} d t^{\prime} \equiv \int_{t^{\prime}=\left(t_{\min }\right)}^{t^{\prime}=t} \rho\left[t^{\prime}\right] d t^{\prime} .
$$

Since Eq. [1.6b] gives $\rho\{t\} \sim\left[1 / t^{2}\right]$, the aim here is to model faster decaying functions such as $\rho\{t\} \sim\left[\exp \left(-\delta_{o} t\right)\right]$ or $\rho\{t\} \sim\left[\exp \left(-q_{o} t^{2}\right)\right]$.

\subsection{Updated Initial Model Results for Italy}

The $Z_{o}[t]$ model of Eq. [1.3a] was applied to bing.com data ${ }^{9}$ for Italy, starting with $N_{\text {data }}^{2 / 23 / 2020}=150$ CoVID-19 cases as an early pandemic point, up through June 15,2020 . Here, $t_{I}$ is when mandatory Social Distancing was introduced at $N_{\text {data }}^{3 / 10 / 2020}=10,149$; with $t_{F}$ being when $N_{\text {data }}^{6 / 15 / 2020}=237,290$. Data prior to Social Distancing $\left(t<t_{I}\right)$ was excluding from this Social Distancing analysis.

Figure 1 compares the $\rho_{\text {data }}[t]$ results with the updated $N_{o}[t]$ and $\rho_{o}[t]=$ $d N_{o}[t] / d t$ predictions using $Z_{o}[t] . \quad$ The $N_{\text {data }}^{2 / 23 / 2020}$ (Day 1$)$ to $N_{\text {data }}^{3 / 10 / 2020}$ (Day 17) interval was examined for estimating a $t=0$ pandemic start where $N_{\text {data }}[t=0] \rightarrow 1$. A best fit value of $t_{\text {of fset }}=9.10055$ days was found, giving $t_{I}=(17-9.10055)=8.89945$ days for $N_{\text {data }}^{3 / 10 / 2020}$ (Day 17), while $N_{\text {data }}^{6 / 15 / 2020}$ (Day 114) gives $t_{F}=(114-9.10055)=104.89945$ days, so that:

$$
\begin{aligned}
& N_{\text {data }}\left[t_{I}=8.899\right]=10,149 ; \\
& N_{\text {data }}\left[t_{F}=104.899\right]=237,290 ; \\
& N_{\text {data }}^{3 / 2020}[t=0]=\mathbf{1}, \\
& N[t \rightarrow \infty]=338,165 ;
\end{aligned}
$$

with $\left\{K_{A}, \gamma_{o}\right\} \approx\{4.2405,0.33078\}$ and $\left(t_{F}-t_{I}\right)=97$ days. In Fig. 1, the X-axis uses this $t=0$ point where $N_{o}[t=0] \rightarrow \mathbf{1}$, and it shows what Social Distancing effects would have been, if it had been operating throughout the $t>0$ period. This $\rho_{o}[t]$ prediction still has a much more gradual drop than the data. This discrepancy indicates that a second Social Distancing process is operating, besides just the gradual $t_{d b l}$ lengthening of the Initial Model.

Figure 2 compares the $N_{o}[t]$ predictions for this model, to the measured $N_{\text {data }}[t]$. Systematic deviations are evident, with the net rms error on a Logarithmic $Y$-axis being $r m s_{\text {error }}=0.097828$. To cure these defects, an enhanced $Z[t]$ model is developed next.

\section{Developing Enhanced $Z[t]$ Models}

To generalize $Z[t]$ beyond Eq. [1.3c], it is convenient to use the $t=\left\{0^{+}, \infty^{-}\right\}$ domain, and require convergence of $Z[t] \rightarrow Z_{o}[t]$ in some limit, along with:

$$
\begin{aligned}
& \operatorname{Lim}_{t \rightarrow 0}\{Z[t]\}=G_{o} \equiv\left[K_{A} / \gamma_{o}\right], \\
& \operatorname{Lim}_{t \rightarrow+\infty}\{Z[t]\}=0,
\end{aligned}
$$

and that the $M_{F}=0$ case of Eq. [1.7a] remains as: 


$$
N(Z)=g_{0} \exp [-Z]
$$

How to choose an appropriate $Z[t]$, as part of an Enhanced Initial Model $(E I M)$, which also allows a $\rho[t] \sim\left[\exp \left(-\delta_{o} t\right)\right]$ or $\rho[t] \sim\left[\exp \left(-q_{o} t^{2}\right)\right]$ stage, is examined next. It can be motivated by studying a simple $N_{T}[t]$ test-case, where $\rho_{T}[t]$ itself is a pure exponential decay, as a function of time:

$$
\begin{aligned}
N_{T}[t] & =\left[e^{+G_{o}}\right]\left[\left(1+e^{-G_{o}}\right)-\exp \left(-\delta_{o} t\right)\right], \\
\rho_{T}[t] & =\left\{d N_{T}[t] / d t\right\}=\left[\delta_{o} e^{+G_{o}}\right] \exp \left(-\delta_{o} t\right),
\end{aligned}
$$

while also preserving $N_{T}\{t \rightarrow 0\}=1$. Comparing Eq. [2.3a] to the Eq. $[2.2]$, sets $Z_{T}[t]$ for this test-case:

$$
\begin{aligned}
& N_{T}[t]=\exp \left[+G_{o}\right] \exp \left(-Z_{T}[t]\right), \\
& Z_{T}[t]=(-1) \ln \left[\left(1+e^{-G_{o}}\right)-\exp \left(-\delta_{o} t\right)\right] .
\end{aligned}
$$

At large times, Eq. $[2.4 \mathrm{~b}]$ gives:

$$
\lim _{t \rightarrow \infty}\left\{Z_{T}[t]\right\} \approx \exp \left(-\delta_{o} t\right)
$$

since $(-\ln [1-x] \approx x)$ for small $x$, which shows that if $\rho_{T}[t]$ has an exponential tail, then $Z_{T}[t]$ also has an exponential tail. A simple generalization for $Z[t]$ in Eq. [1.7a]-[1.7d] would be either:

$$
\begin{aligned}
& Z_{E}[t] \equiv+\left[G_{o} /\left(1+\gamma_{o} t\right)\right]\left[\exp \left(-\delta_{o} t-q_{o} t^{2}\right)\right], \\
& Z_{A}[t] \equiv+\left[G_{o} /\left(1+\gamma_{o} t\right)\right]\left[\exp \left(-\delta_{o} t\right)\right]=Z_{E}\left[t ; q_{o} \equiv 0\right] .
\end{aligned}
$$

As with Eq. [1.3c], the original CoVID-19 exponential growth factor $K_{A}$ remains only as part of the $G_{o}$ scaling factor, while the $\left\{\delta_{o} \rightarrow 0\right\}$ limit of Eq. [2.6b] converges back to the Eq. [1.3c] Initial Model.

Using Eq. [2.6a] for $Z_{E}[t]$ in the Eq. [2.2] $N\left(Z_{E}\right)$ example gives:

$$
\begin{aligned}
\rho[t]= & \{d N[t] / d t\}=g_{0} \frac{d}{d t} \exp \left[-Z_{E}\right]=-g_{0} \exp \left[-Z_{E}\right] \frac{d}{d t} Z\left[t_{E}\right]= \\
& +N\left(Z_{E}\right)\left[\exp \left(-\delta_{o} t-q_{o} t^{2}\right)\right]\left\{\frac{+G_{o} \gamma_{o}}{\left(1+\gamma_{o} t\right)^{2}}+\frac{G_{o}}{\left(1+\gamma_{o} t\right)}\left[+\delta_{o}+2 q_{o} t\right]\right\},
\end{aligned}
$$

which exhibits the following variety of long-time limits:

$$
\begin{aligned}
& \rho\left[t ; \delta_{o}=0, q_{o}=0\right] \rightarrow N\left(Z_{E}\right)\left\{\frac{+G_{o} \gamma_{o}}{\left(1+\gamma_{o} t\right)^{2}}\right\}, \\
& \rho\left[t ; q_{o}=0\right] \rightarrow N\left(Z_{E}\right)\left[\exp \left(-\delta_{o} t\right)\right]\left\{\frac{+G_{o} \delta_{o}}{\left(1+\gamma_{o} t\right)}\right\}, \\
& \rho\left[t ; \delta_{o}=0\right] \rightarrow N\left(Z_{E}\right)\left[\exp \left(-q_{o} t^{2}\right)\right]\left\{\frac{2 G_{o} q_{o}}{\gamma_{o}}\right\}, \\
& \rho[t] \rightarrow N\left(Z_{E}\right)\left\{\exp \left[-q_{o}\left(t+\frac{\delta_{o}}{2 q_{o}}\right)^{2}\right]\right\}\left\{\frac{2 G_{o} q_{o}}{\gamma_{o}} \exp \left[+\frac{1}{4} \delta_{o}^{2} / q_{o}\right]\right\} .
\end{aligned}
$$

Here, any $q_{o} \neq 0$ Gaussian component in $Z_{E}[t]$ gives a $\rho[t]$ tail that is also a pure Gaussian. An exponential component $\left(q_{o} \equiv 0\right)$ in $Z_{E}[t]$ gives a timemodified exponential $\rho[t]$ tail, while having $\left\{q \equiv 0, \delta_{o} \equiv 0\right\}$ in $Z_{E}[t]$ gives the prior $\rho[t]^{\sim}\left(1 / t^{2}\right)$ result of Eq. [1.6b].

\section{Pandemic Fast vs Slow Shutoffs}

Each $Z_{E}[t]$ function modifies $N[t]$ predictions for the pandemic start, pandemic end, and the mid-range where $\rho[t]$ has its pandemic peak. The Eq. [2.6a] $Z_{E}[t]$ function especially alters the calculated CoVID-19 pandemic tail. For either $q_{o} \neq 0$ or $\delta_{o} \neq 0$, Eqs. [2.6a]-[2.6b] gives a pandemic Fast Shutoff, compared to the gradually decreasing $Z_{o}[t]$ of Eq. $[1.3 \mathrm{c}]$ in the Initial Model, which is a pandemic Slow Shutoff. However $Z_{o}[t]$ from the Initial Model, and $Z_{A}[t]$ from Eq. [2.6b] both gave long-term $\rho[t]$ tails that decay much slower than the $q_{o} \neq 0$ Eq. [2.6a] Gaussian. 
If data does not show evidence of a Gaussian pandemic Fast Shutoff, assuming the post-peak $\rho[t]$ data will be Gaussian is likely to provide optimistically inaccurate $N[t]$ predictions for CoVID-19 pandemic evolution. Apparently, this is exactly what was done by the University of Washington IHME (Institute of Health Metrics and Evaluation) in their widely publicized initial preprint ${ }^{3}$ of 27 March 2020, with this Gaussian model continuing throughout their subsequent updates $^{4-6}$ up through 29 April 2020.

IHME changed everything in their 4 May $2020^{7-8}$ update. They no longer used $\rho[t]$ Gaussian tails, and it doubled or tripled their predicted CoVID-19 pandemic death rates. Thus, unless the post-peak $\rho[t]$ exhibits Gaussian behavior, the $Z_{A}[t]$ with $\delta_{o} \neq 0$ is likely the most important modification to $Z_{o}[t]$, which is the pandemic Fast Shutoff model used here.

Using $Z_{A}[t]$, the pandemic Fast Shutoff can be extrapolated to calculate a pandemic start point where $N[t=0]=\mathbf{1}$. We then examine if this $Z_{A}[t]$ must also carry over to the $\rho[t]$ long-term tail,

Since the long-term low $\rho_{\text {data }}[t]$ tail may differ among localities, and is not well known, the $\delta_{o} \neq 0$ case of Eq. [2.6b] could end with a Slow Shutoff, giving:

$$
\begin{aligned}
& Z_{B}[t]=+\left[G_{o} /\left(1+\gamma_{o} t\right)\right]\left\{\exp \left[-\delta_{o} t /\left(1+\lambda_{o} t\right)\right]\right\}, \\
& \lim _{t \rightarrow \infty}\left\{Z_{B}[t]\right\} \approx+\left[G_{o} /\left(1+\gamma_{o} t\right)\right]\left\{\exp \left[-\delta_{o} / \lambda_{o}\right]\right\},
\end{aligned}
$$

with $G_{o}$ as in Eq. [2.1a]. This Eq. [3.1a] $Z_{B}[t]$ function has the $\left\{\delta_{o}, \lambda_{o}\right\}$ Mitigation Measure operating at the start of Social Distancing, but reverting to the Initial Model in the long-time limit. Combining Eq. [2.6b] and Eq. [3.1a] cases gives this Enhanced Initial Model (EIM) equation:

$$
Z_{B}[t]=+\left[G_{o} /\left(1+\gamma_{o} t\right)\right]\left\{\exp \left[-\delta_{o} t /\left(1+\kappa \gamma_{o} t\right)\right]\right\},
$$

where $\kappa=0$ is a pure exponential, and $\kappa=1$ has a modified tail that includes its own long-term shutoff. Comparing $\kappa=\{0,1\}$ in Eq. [3.2] provides a simple test for which model matches CoVID-19 data better in any locality. Any other $\kappa>0$ value then recovers the more general $\lambda_{o} \equiv \kappa \gamma_{o}$ case. This Eq. [3.2] $Z_{B}[t]$ replaces $Z_{o}[t]$ of Eq. [1.3c], and its EIM companion $N_{B}[t]$ is:

$$
N_{B}[t]=\left[e^{+G_{o}}\right]\left[\exp \left(-Z_{B}[t]\right),\right.
$$

while using $Z[t] \rightarrow Z_{B}[t]$ in Eqs. [1.7a]-[1.7d] gives an Enhanced Orthogonal Function $[E O F]$ model.

\section{Finding $\left\{K_{A}, \gamma_{o}, \delta_{o}\right\}$ for $Z_{B}[t]$ from Data}

If $\delta_{o}=0$, the prior Eqs. [1.4a]-[1.5b] for $\left\{K_{A}, \gamma_{o}, \delta_{o}=0\right\}$ and $\left\{t_{I}, t_{F}\right\}$ can be used, with the initial best-fit $\left\{K_{o}, \alpha_{S}\right\}$ values determined by minimizing the rms error between Eq. [1.2] and the measured data on a Logarithmic Y-axis. Unfortunately, Eqs. [1.4a]-[1.5b] cannot be used when $\delta_{o} \neq 0$, although finding a good $\left\{K_{A}, \gamma_{o}, \delta_{o}\right\}$ starting point is still needed for the EIM:

$$
Z_{B}[t]=+\left[G_{o} /\left(1+\gamma_{o} t\right)\right]\left\{\exp \left[-\delta_{o} t /\left(1+\kappa \gamma_{o} t\right)\right]\right\},
$$

prior to any $E O F$ analysis. The $\kappa=1$ case also has this special symmetry:

$$
\begin{aligned}
& {\left[+\delta_{o} t /\left(1+\gamma_{o} t\right)\right]=\left(\delta_{o} / \gamma_{o}\right)\left[1-\frac{1}{\left(1+\gamma_{o} t\right)}\right],} \\
& \exp \left[-\delta_{o} t /\left(1+\gamma_{o} t\right)\right]=e^{-\left(\delta_{o} / \gamma_{o}\right)} \exp \left[+\left(\delta_{o} / \gamma_{o}\right) \frac{1}{\left(1+\gamma_{o} t\right)}\right],
\end{aligned}
$$




$$
\begin{aligned}
& Z_{B}[t]=+\left[\frac{G_{o}}{\left(1+\gamma_{o} t\right)}\right] e^{-\left(\delta_{o} / \gamma_{o}\right)} \exp \left[+\left(\delta_{o} / \gamma_{o}\right) \frac{1}{\left(1+\gamma_{o} t\right)}\right], \\
& Z_{B}[t]=+G_{o}\left[\frac{\left(\delta_{o} / \gamma_{o}\right)}{\left(1+\gamma_{o} t\right)}\right] \frac{1}{\left(\delta_{o} / \gamma_{o}\right) e^{+\left(\delta_{o} / \gamma_{o}\right)}} \exp \left[+\left(\delta_{o} / \gamma_{o}\right) \frac{1}{\left(1+\gamma_{o} t\right)}\right],
\end{aligned}
$$

which can re-written as:

$$
\begin{aligned}
& W(X[t]) \equiv X[t] \exp (+X[t]), \\
& X[t]=\left[\left(\delta_{o} / \gamma_{o}\right) /\left(1+\gamma_{o} t\right)\right], \\
& X[0]=\left[\delta_{o} / \gamma_{o}\right], \\
& Z_{B}[t]_{\kappa=1}=+G_{o} W(X[t]) / W(X[0]) . \\
& N_{B}\left(Z_{B}\right)=\left[e^{+G_{o}}\right] \exp \left(-Z_{B}[t]_{\kappa=1}\right),
\end{aligned}
$$

$$
\begin{aligned}
& Z_{A}[t]=+\left[G_{o} /\left(1+\gamma_{o} t\right)\right] \exp \left(-\delta_{o} t\right), \\
& N_{A}\left(Z_{A}\right)=\left[e^{+G_{o}}\right] \exp \left(-Z_{B}[t]_{\kappa=0}\right)=\left[e^{+G_{o}}\right] \exp \left(-Z_{A}[t]\right) .
\end{aligned}
$$

For $\kappa=\{0,1\}$, the $t=0$ point, $G_{O}$ from Eq. [2.1a], and the $N_{I}\left(t_{I}\right)$ and $N_{F}\left(t_{F}\right)$ initial and final points, give these equations to help set $\left\{K_{A}, \gamma_{o}, \delta_{o}\right\}$ :

$$
\begin{aligned}
& N_{I}[t=0]=\mathbf{1}=G_{O} \exp \left(-Z_{B}[t=0]\right), \\
& N_{I}\left[t_{I}\right]=N_{I}=G_{o} \exp \left(-Z_{B}\left[t_{I}\right]\right), \\
& N_{F}\left[t_{F}\right]=N_{F}=G_{O} \exp \left(-Z_{B}\left[t_{F}\right]\right),
\end{aligned}
$$

for the EIM. Minimizing the rms error between the Eq. [3.3] $\left\{K_{A}, \gamma_{o}, \delta_{o}, t_{I}\right\}$ functions and measured data on a Logarithmic $Y$-axis can be done as follows. Start with estimated values for $\left\{\widehat{K}_{A}, \widehat{\gamma}_{o}, \widehat{\delta}_{o}, \widehat{t}_{\text {offset }}\right\}$ in:

$$
\begin{aligned}
& \widehat{Z}[t]=+\left[\widehat{G}_{o} /\left(1+\widehat{\gamma}_{o} t\right)\right]\left\{\exp \left[-\widehat{\delta}_{o} t /\left(1+\kappa \widehat{\gamma}_{o} t\right)\right]\right\}, \\
& \widehat{N}[t]=\left[e^{+\widehat{G}_{o}}\right][\exp (-\widehat{Z}[t]), \\
& \widehat{G}_{o} \equiv\left(\widehat{K}_{A} / \widehat{\gamma}_{o}\right) \\
& t=\left(t_{\text {data }}-\widehat{t}_{\text {offset }}\right),
\end{aligned}
$$

where $t_{\text {data }}$ is the data start time. Set a preliminary value for $\widehat{t}_{\text {offset }}$ first, to fix the time scale for the $N_{\text {data }}[t]$ measured values:

$$
\begin{aligned}
& N_{I} \equiv N_{\text {data }}\left[t_{\text {data }}^{I}-\widehat{t}_{\text {offset }}\right], \\
& N_{F} \equiv N_{\text {data }}\left[t_{\text {data }}^{F}-\widehat{t}_{\text {offset }}\right], \\
& \left(t_{F}-t_{I}\right) \equiv\left(t_{\text {data }}^{F}-t_{\text {data }}^{I}\right) .
\end{aligned}
$$

Next, pick values for $\left\{\widehat{\gamma}_{o}, \widehat{\delta}_{o}\right\}$ for $\widehat{N}[t]$ in Eq. [4.6b], allowing direct comparison between $N[t]$ and $\widehat{N}[t]$ at each data point:

$$
\begin{aligned}
& \widehat{N}[t] \equiv \widehat{N}\left[t=\left(t_{\text {data }}-\widehat{t}_{\text {offset }}\right)\right] \\
& N_{\text {data }}[t] \equiv N_{\text {data }}\left[t=\left(t_{\text {data }}-\widehat{t}_{\text {offset }}\right)\right] .
\end{aligned}
$$

The resulting calculated values for both $\left\{\widehat{N}\left[t_{I}\right], \widehat{N}\left[t_{F}\right]\right\}$ can often be much too high or low, compared to the $\left\{N_{I}, N_{F}\right\}$ measured data, but those values can be renormalized to:

$$
\begin{aligned}
& \bar{N}[t]=\widehat{N}[t]\left(N_{I} / \widehat{N}\left[t_{I}\right]\right) \equiv S_{I} \widehat{N}[t], \\
& \bar{N}\left[t_{F}\right]=\widehat{N}\left[t_{F}\right]\left(N_{I} / \widehat{N}\left[t_{I}\right]\right) \equiv S_{I} \widehat{N}\left[t_{F}\right] .
\end{aligned}
$$

Here, $S_{I}$ is the renormalization coefficient, and $\bar{N}[t]$ allows easy comparison to the measured $N_{\text {data }}[t]$ since $\bar{N}\left[t_{I}\right] \equiv N_{I}$. Given $\left\{\widehat{\gamma}_{o}, \widehat{\delta}_{o}\right\}$, the $\widehat{K}_{A}\left(\widehat{\gamma}_{o}\right)$ value that is needed to obey $\bar{N}\left[t_{F}\right] \rightarrow N_{F}$ can be set by using Excel ${ }^{T M}$ _Goal-Seek or its equivalent, which also sets a particular $S_{I}$ value. Next, the $\widehat{\gamma}_{o}$ value is adjusted to find the specific $\left\{K_{A}, \gamma_{o}\right\}$ parameter pair that gives $S_{I}=1$. This process is needed because these $\widehat{\delta}_{o} \neq 0$ cases do not allow easy determination of $t_{I}$ as in Eq. [1.4a], or for $\left\{K_{A}, \gamma_{o}\right\}$, given $t_{I}$, as in Eqs. [1.5a]-[1.5b]. 
The rms error on a Logarithmic $Y$-axis, between this $\bar{N}\left[t ; K_{A}, \gamma_{o}\right]$ and the $N_{\text {data }}[t]$ is one of many $\left\{K_{A}, \gamma_{o}, \widehat{\delta}_{o}, \widehat{t}_{\text {offset }} ; S_{I}=1\right\}$ choices. The minimum rms error among all these $S_{I}=1$ cases and the $N_{\text {data }}[t]$, when varying $\left\{\widehat{\delta}_{o}, \widehat{t}_{\text {offset }}\right\}$ gives the best $\left\{K_{A}, \gamma_{o}, \delta_{o}, t_{o f f s e t}\right\}$ values for Eqs. [4.3a]-[4.3e].

\section{$5 \quad$ Enhanced Initial Model [EIM] Results for Italy}

The Eq.[1.3a] Initial Model results were shown in Figs. 1-2. Mandatory Social Distancing was introduced at $N_{\text {data }}^{3 / 10 / 2020}=10,149$ which is the $t_{I}$ data point, with $N_{\text {data }}^{6 / 15 / 2020}=237,290$ being the $t_{F}$ data point. The $E I M$ was then applied to the same data, to highlight the improvements that can be obtained from using the $E I M$ of $Z_{A}[t]$ and $N_{A}\left(Z_{A}\right)$, in place of $Z_{o}[t]$ and $N_{o}\left(Z_{o}\right)$.

Figures 3-4 show the resulting $E I M$ best-fits for $Z_{A}[t]$ and $N_{A}\left(Z_{A}\right)$, along with using a $\rho_{A}[t]$ tail that is a pure exponential decay.

For the EIM, a new best estimate of $t_{\text {offset }}=2.866$ days was found within the $N_{\text {data }}^{2 / 23 / 2020}$ (Day 1) to $N_{\text {data }}^{3 / 10 / 2020}$ (Day 17) data, setting the EIM $t=0$ point. Then $t_{I}=(17-2.866)=14.134$ days, while $N_{\text {data }}^{6 / 15 / 2020}$ (Day 114) gives $t_{F}=(114-2.866)=111.134$ days, along with:

$$
\begin{aligned}
& N_{\text {data }}^{3 / 10 / 2020}\left[t_{I}=14.134\right]=10,149 ; \\
& N_{\text {data }}^{6 / 15 / 2020}\left[t_{F}=111.134\right]=237,290 ; \\
& N_{\text {data }}^{2 / 25 / 2020}[t=0]=\mathbf{1}, \\
& \left(t_{F}-t_{I}\right)=97 \text { days } .
\end{aligned}
$$

This X-axis $t=0$ point is a hypothetical EIM pandemic starting point, if Social Distancing had been operating throughout the initial CoVID-19 period. Figure 3 has a predicted CoVID-19 pandemic peak of $\sim 5,217 /$ day at $t=$ 29.134 days on $3 / 25 / 2020$, with $\sim 243,100$ total cases at the pandemic end. This datafit has $>4 X$ error reduction over the $\delta_{o}=0$ case, as summarized next:

$$
\begin{array}{ccccccc}
K_{A} & \gamma_{o} & t_{d b l}, \text { days } & N[t \rightarrow \infty] & \delta_{o} & t_{I}, \text { days } & r m s_{E R R} \\
1.285436 & .101062 & .553060 & \sim 243,100 & .0336 & 14.134 & 0.023755 \\
4.240513 & .33078 & .163458 & \sim 338,165 & \mathbf{0} & 7.899 & 0.097828
\end{array}
$$

The EIM with $Z_{A}[t]$ and $\delta_{o}=0.0336$ gives a $\rho_{A}[t]$ curve that is in excellent agreement with the Fig. 3 measured $\rho_{\text {data }}[t]$ data. Comparing $N_{A}[t]$ and $N_{\text {data }}[t]$ in Fig. 4 also shows an excellent match over the whole Logarithmic $Y$-axis range, used in the rms error minimization.

The Figs. 3-4 $\kappa=0$ results were then compared to the $\kappa=1$ case, using $Z_{B}[t]$ and $N_{B}\left(Z_{B}\right)$ of Eqs. [3.2]-[3.3]. The $\kappa=1$ case has a Social Distancing factor that gradually turns off the EIM exponential decay. The resulting rms error best fits converged to $\delta_{o} \rightarrow 0$, as follows:

$\begin{array}{cccccc}\gamma_{o} & t_{d b l} & r m s_{E R R} & \delta_{o} & t_{\text {offset }}^{\text {days }} & t_{I}^{\text {days }}:: t_{F}^{\text {days }} \\ .07146 & .775863 & .2223783 & .2000 & 10.0 & 7:: 104 \\ .18623 & .295372 & .1751635 & .2000 & 11.0 & 6:: 103 \\ .30360 & .181417 & .2102839 & .2000 & 12.0 & 5:: 102\end{array}$




$\begin{array}{llllllll}.39748 & .137925 & .1430275 & .0500 & 11.0 & 6:: 103 & \\ .43604 & .125722 & .1425737 & .0135 & 11.0 & 6:: 103 & \\ .44265 & .123844 & .1425524 & .0070 & 11.0 & 6:: 103 & \\ .44466 & .123284 & .1425480 & .0050 & 11.0 & 6:: 103 & \\ .44868 & .122179 & .1425445 & .0010 & 11.0 & 6:: 103 & \\ .44968 & .121907 & .1425442 & .0000 & 11.0 & 6:: 103 . & {[5.3]}\end{array}$

This $\kappa=1$ result shows that the process giving rise to exponential tails in the $\rho_{\text {data }}[t]$ for a pandemic Fast Shutoff is independent of the Social Distancing process that gradually lengthens the $t_{d b l}$ doubling time, as measured by $\gamma_{o}$. Comparing the $\kappa=\{0,1\}$ cases shows that the pure exponential tail with $Z_{A}[t]=Z_{B}[t]_{\kappa=0}$ matches the $\rho_{\text {data }}[t]$ data best.

\section{Enhanced Orthogonal Functions for Italy}

Any monotonic $Z[t]$ can convert measured $N_{\text {data }}[t]$ data into $N_{\text {data }}(Z)$. Using the Eq. [2.6b] $Z_{A}[t] \rightarrow Z[t]$ in Eqs. [1.7a]-[1.7d] extends the $E I M$ into an $E O F$ model, where Eq. [1.9] gives:

$$
\begin{aligned}
& \rho[t]=R\left(Z_{A}[t]\right) \frac{d Z_{A}}{d t}=Z_{A}[t] R\left(Z_{A}[t]\right)\left\{\delta_{o}+\frac{\gamma_{o}}{\left(1+\gamma_{o} t\right)}\right\}, \\
& Z_{A}[t]=+\left[G_{o} /\left(1+\gamma_{o} t\right)\right] \exp \left(-\delta_{o} t\right) .
\end{aligned}
$$

The Eq. [5.2] $\delta_{o} \neq 0$ entries and Eq. [5.1a]-[5.1d] boundary conditions give:

$$
\begin{array}{ll}
Z_{A}^{\min }\left[t_{F}=111.134\right]=0.024226510, & {[6.2 \mathrm{a}]} \\
Z_{A}^{\max }\left[t_{I}=14.134\right]=3.176125728 . & {[6.2 \mathrm{~b}]}
\end{array}
$$

These data-driven $Z_{A}[t]$ limits are used next, along with $Z[t] \rightarrow Z_{A}[t]$ in:

$$
\begin{aligned}
& N(Z)= \sum_{m=0}^{m=M_{F}} g_{m} L_{m}(Z) \exp [-Z], \\
& g_{n} \equiv \sum_{m=0}^{m=M_{F}} g_{m} \int_{Z=+\infty}^{Z=+\infty} L_{n}(Z) L_{m}(Z) \exp [-Z] d Z= \\
& \int_{Z=Z_{A}^{\max }}^{\min } L_{n}(Z) N_{\text {data }}(Z) d Z+ \\
& Z=+\infty \int_{Z=Z_{A}^{\max }}^{\max } L_{n}(Z) N(Z) d Z+\int_{Z=0}^{Z=Z_{A}^{\min }} L_{n}(Z) N(Z) d Z,
\end{aligned}
$$

where $m=\left\{0, M_{F}\right\}$ sets how many terms are in the Eq. [6.3a] series. Generally $M_{F}=2$ is used here. The $L_{m}(Z)$ are the Laguerre Polynomials, with the first few $L_{m}(Z)$ being:

$$
\begin{aligned}
& L_{-1}(Z) \equiv 0 \\
& L_{0}(Z) \equiv 1=L_{m}(Z=0) \\
& L_{1}(Z) \equiv(1-Z) \\
& L_{2}(Z) \equiv\left(1-2 Z+\frac{1}{2} Z^{2}\right) \\
& L_{3}(Z) \equiv\left(1-3 Z+\frac{3}{2} Z^{2}-\frac{1}{6} Z^{3}\right) \\
& L_{4}(Z) \equiv\left(1-4 Z+3 Z^{2}-\frac{2}{3} Z^{3}+\frac{1}{24} Z^{4}\right) .
\end{aligned}
$$

Some important properties of the Laguerre Polynomials are:

$$
\int_{Z=0}^{Z=+\infty} L_{m}(Z) L_{n}(Z) \exp (-Z) d Z=\mathbf{1} \delta_{m, n},
$$




$$
\begin{aligned}
& \delta_{m, n}=\left(\begin{array}{lll}
\mathbf{1} & \text { for } & m=n \\
\mathbf{0} & \text { otherwise }
\end{array}\right), \\
& \int_{Z^{\prime}=Z}^{Z^{\prime}=+\infty} L_{m}\left(Z^{\prime}\right) \exp \left(-Z^{\prime}\right) d Z^{\prime}=\left[L_{m}(Z)-L_{m-1}(Z)\right] \exp (-Z), \\
& L_{m}(Z) \exp (-Z)=\frac{1}{m !} \frac{d^{m}}{d Z^{m}}\left[Z^{m} e^{-Z}\right]=e^{-Z^{k=m}} \sum_{k=0}^{k=1}(-1)^{k} \frac{m !}{k !(m-k) !}\left[\frac{Z^{k}}{k !}\right], \\
& L_{m \geq 2}(Z)=\left[2-\frac{(Z+1)}{m}\right] L_{m-1}(Z)-\left[1-\frac{1}{m}\right] L_{m-2}(Z) .
\end{aligned}
$$

Here Eq. [6.5a] defines an orthogonal function set. The "n!" (n-factorial) in Eq. [6.5d], for $\mathbf{n}$ an integer, is defined as the product:

$$
\begin{array}{lr}
\mathbf{n} ! \equiv(\mathbf{n})(\mathbf{n}-1)(\mathbf{n}-2)(\mathbf{n}-3) \ldots(3)(2)(1), & {[6.6 \mathrm{a}]} \\
\mathbf{1} ! \equiv \mathbf{0} ! \equiv 1, & {[6.6 \mathrm{~b}]}
\end{array}
$$

where factorials with negative integers are not allowed. For $M_{F}>2$, the following equations developed by Watson ${ }^{\mathbf{1 0}}$, and improved by Gillis and Weiss ${ }^{\mathbf{1 1}}$, helps in evaluating Eq. [6.3b]:

$$
\begin{aligned}
& L_{r}(Z) L_{s}(Z)=\sum_{t=|r-s|}^{t=(r+s)} C_{r s t} L_{t}(Z), \\
& C_{r s t}=\int_{X=0}^{X=+\infty} L_{r}(X) L_{s}(X) L_{t}(X) \exp (-X) d X, \\
& C_{r s t} \equiv \frac{(-1)^{p}}{2^{p}} \sum_{n=0}^{n=(r+s)}\left(2^{2 n}\right) \frac{(r+s-n) !}{(r-n) !(s-n) !(2 n-p) !(p-n) !}, \\
& p \equiv(r+s-t),
\end{aligned}
$$

where ALL terms in the Eq. [6.7c] sum for $n=\{0,(r+s)\}$ have an implicit requirement that all negative factorial arguments are excluded ${ }^{\mathbf{1 0}-\mathbf{1 1}}$.

Since the $N(Z)$ of Eq. [6.3a] has $\left\{g_{m} ; m=\left(0, M_{F}\right)\right\}$, and $N(Z)$ also appears in each $g_{n}$-equation of Eq. [6.3b], how to determine each $g_{m}$ by itself, can be done as follows. First define:

$$
\begin{aligned}
& Q_{n} \equiv \int_{Z=Z_{A}^{\min }}^{Z=Z_{A}^{\max }} L_{n}(Z) N_{\text {data }}(Z) d Z \\
& K_{m, n} \equiv \int_{Z=Z_{A}^{\min }}^{Z=Z_{A}^{\max }} L_{m}(Z) L_{n}(Z) \exp (-Z) d Z=K_{n, m} .
\end{aligned}
$$

When the $N_{\text {data }}(Z)$ is comprised of $j=\{1,2, \ldots J\}$ discrete values between $\left\{Z_{A}^{\min }, Z_{A}^{\max }\right\}$, with each $Z_{j}$ having an $N_{\text {data }}^{(j)}\left(Z_{j}\right)$ value, the Eq. [6.8a] integral needs to be replaced by a sum. Let $Z_{0}=Z_{1}$ and $Z_{J+1}=Z_{J}$, the $Q_{n}$ replacement for Eq. [6.8a] is then:

$$
\begin{aligned}
Q_{n} & \equiv \sum_{j=1}^{j=J} L_{n}\left(Z_{j}\right) N_{d a t a}^{(j)}\left(Z_{j}\right) \Delta_{j}, \\
\Delta_{j} & \equiv \frac{1}{2}\left|Z_{j+1}-Z_{j-1}\right| .
\end{aligned}
$$

Eq. $[6.3 \mathrm{~b}]$ can then be re-written as a $3 \times 3$ matrix $\underline{\mathbf{M}}_{3}$, which relates a data-driven $\vec{Q}_{3}$-vector to a resultant $\vec{g}_{3}$-vector:

$$
\left.\begin{array}{c}
\vec{Q}_{3}=\underline{\mathbf{M}}_{3} \vec{g}_{3} \\
Q_{0} \\
Q_{2}
\end{array}\right)=\left(\begin{array}{lll}
K_{0,0} & K_{0,1} & K_{0,2} \\
K_{1,0} & K_{1,1} & K_{1,2} \\
K_{2,0} & K_{2,2} & K_{2,2}
\end{array}\right)\left(\begin{array}{l}
g_{0} \\
g_{1} \\
g_{2}
\end{array}\right) .
$$




$$
\left(\underline{\mathbf{M}}_{3}\right)^{-1} \vec{Q}_{3} \equiv \vec{g}_{3},
$$

where $\left(\underline{\mathbf{M}}_{3}\right)^{-\mathbf{1}}$ is the matrix inverse of $\underline{\mathbf{M}}_{3}$. When $\left\{Z_{A}^{\min }, Z_{A}^{\max }\right\} \rightarrow\{0,+\infty\}$, this $\underline{\mathbf{M}}_{3}$ becomes the Identity Matrix. The following $k_{m, n}(Z)$ integrals set $K_{m, n}$ :

$$
\begin{aligned}
& k_{m, n}(Z)=\int_{Z^{\prime}=Z}^{Z^{\prime}=+\infty} L_{m}\left(Z^{\prime}\right) L_{n}\left(Z^{\prime}\right) \exp \left(-Z^{\prime}\right) d Z^{\prime}=k_{n, m}(Z), \\
& K_{m, n} \equiv k_{m, n}\left(Z_{A}^{\min }\right)-k_{m, n}\left(Z_{A}^{\max }\right)=K_{n, m} .
\end{aligned}
$$

The $k_{m, n}(Z)$ integrals can be determined using Eq. [6.5c], which gives:

$$
\begin{aligned}
& k_{0,0}(Z)=1 \exp (-Z), \\
& k_{1,1}(Z)=\left\{1+Z^{2}\right\} \exp (-Z), \\
& k_{2,2}(Z)=\left\{1+2 Z^{2}-Z^{3}+\frac{1}{4} Z^{4}\right\} \exp (-Z), \\
& k_{0,1}(Z)=(-Z) \exp (-Z), \\
& k_{0,2}(Z)=(-Z)\left\{1-\frac{1}{2} Z\right\} \exp (-Z), \\
& k_{1,2}(Z)=(-Z)\left\{1-Z+\frac{1}{2} Z^{2}\right\} \exp (-Z) .
\end{aligned}
$$

To extract $\left\{g_{0}, g_{1}, g_{2}\right\}$, the $3 \times 3$ symmetric $\underline{\mathbf{M}}_{3}$ matrix needs inversion:

$$
\begin{aligned}
& \underline{\mathbf{M}}=\left(\begin{array}{ccc}
a & d & f \\
d & b & e \\
f & e & c
\end{array}\right), \\
& \operatorname{det}[\underline{\mathbf{M}}] \equiv\left[a b c-a e^{2}-b f^{2}-c d^{2}+2 d e f\right], \\
& \operatorname{det}[\underline{\mathbf{M}}](\underline{\mathbf{M}})^{-\mathbf{1}} \equiv\left(\begin{array}{ccc}
{\left[b c-e^{2}\right]} & -(c d-e f) & -(b f-d e) \\
-(c d-e f) & {\left[a c-f^{2}\right]} & -(a e-d f) \\
-(b f-d e) & -(a e-d f) & {\left[a b-d^{2}\right]}
\end{array}\right),
\end{aligned}
$$

which determines $\left\{g_{0}, g_{1}, g_{2}\right\}$ from the $\left\{Q_{0}, Q_{1}, Q_{2}\right\}$ data. A best-fit $N(Z)$ for $Z=\left\{0^{+}, \infty^{-}\right\}$results, along with an equivalent fit for $R(Z)$ using Eq. [1.7d].

Instead of having to find the best $\left\{g_{0}, g_{1}, g_{2}\right\}$ triplet, one could find the best $\left\{g_{0}^{\prime}, g_{1}^{\prime}\right\}$ by just using using $\left\{Q_{0}, Q_{1}\right\}$ and an $\underline{\mathbf{M}}_{2}$ sub-matrix; or one could find the best $\left\{g_{0}^{+}\right\}$by itself by just using $\left\{Q_{0}\right\}$ and an $\underline{\mathbf{M}}_{1}$ sub-matrix:

$$
\begin{aligned}
& \vec{Q}_{2}=\underline{\mathbf{M}}_{2} \vec{g}_{2} \text {, } \\
& \left(\begin{array}{l}
Q_{o} \\
Q_{1}
\end{array}\right)=\left(\begin{array}{ll}
K_{0,0} & K_{0,1} \\
K_{1,0} & K_{1,1}
\end{array}\right)\left(\begin{array}{c}
g_{0}^{\prime} \\
g_{1}^{\prime}
\end{array}\right), \\
& \left(\begin{array}{c}
g_{0}^{\prime} \\
g_{1}^{\prime}
\end{array}\right)=\left(\begin{array}{ll}
K_{0,0} & K_{0,1} \\
K_{1,0} & K_{1,1}
\end{array}\right)^{-1}\left(\begin{array}{c}
Q_{o} \\
Q_{1}
\end{array}\right)= \\
& \frac{1}{\left[K_{0,0} K_{1,1}-K_{0,1} K_{1,0}\right]}\left(\begin{array}{cc}
K_{1,1} & K_{1,0} \\
K_{0,1} & K_{0,0}
\end{array}\right)\left(\begin{array}{c}
Q_{o} \\
Q_{1}
\end{array}\right), \\
& \vec{Q}_{1}=\underline{\mathbf{M}}_{1} \vec{g}_{1} \text {, } \\
& \left(Q_{o}\right)=\left(K_{0,0}\right)\left(g_{o}^{+}\right) \text {, } \\
& \left(g_{o}^{+}\right)=\left(K_{0,0}\right)^{-1}\left(Q_{o}\right) \text {. }
\end{aligned}
$$

Once the $\left\{g_{m} ; m=\left(0, M_{F}\right)\right\}$ constants are found and used in Eq. [1.8], its $c_{0}$ value provides the new EOF estimate for the predicted total number of CoVID-19 cases at the pandemic end, refining the initial Eq. [5.2] $N[t \rightarrow \infty]$ EIM value. 


\section{$7 \quad$ EOF Model Results for Italy}

The EOF model starts with the EIM of Eq. [2.6b] using $Z_{A}[t]$, and the bing.com Italy data ${ }^{9}$, which gives:

$$
\begin{aligned}
& N_{\text {data }}\left[t_{I}=14.134\right]=10,149 ; \\
& N_{\text {data }}\left[t_{F}=111.134\right]=237,290 ; \\
& N_{A}[t \rightarrow \infty]=243,109 \\
& Z_{A}^{\min }\left[t_{F}=111.134\right]=0.024226510, \\
& Z_{A}^{\max }\left[t_{I}=14.134\right]=3.176125728,
\end{aligned}
$$

via Eqs. [5.1a]-[5.1d], [5.2], and [6.2a]-[6.2b]. For these $\left\{Z_{A}^{\min }, Z_{A}^{\max }\right\}$ values, with $\left(t_{F}-t_{I}\right)=97$ days, the $\underline{\mathbf{M}}_{3}$ matrix of $K_{m, n}$ entries, via Eq. [6.8b], is:

$$
\underline{\mathbf{M}}_{3}=\left(\begin{array}{lll}
K_{0,0} & K_{0,1} & K_{0,2} \\
K_{1,0} & K_{1,1} & K_{1,2} \\
K_{2,0} & K_{2,2} & K_{2,2}
\end{array}\right)=\left(\begin{array}{ccc}
0.93432 & 0.10895 & -0.10133 \\
0.10895 & 0.51376 & 0.35717 \\
-0.10133 & 0.35717 & 0.36868
\end{array}\right)
$$

It has a rather small $\operatorname{det}\left[\underline{\mathbf{M}}_{3}\right]=0.04024218$ value, with an inverse of:

$$
\left(\underline{\mathbf{M}}_{3}\right)^{-1} \equiv\left(\begin{array}{ccc}
1.536745 & -1.897502 & 2.260643 \\
-1.897502 & 8.304570 & -8.566823 \\
2.260643 & -8.566823 & 11.633092
\end{array}\right)
$$

A convolution of $L_{m}\left(Z_{A}\right)$ functions with the measured $\vec{Q}_{3}$ dataset vector of Eqs. [6.9a]-[6.9b], along with the above $\left(\underline{\mathbf{M}}_{3}\right)^{-\mathbf{1}}$, gives this final $\vec{g}$-vector ${ }^{\mathbf{1 2}}$ :

$$
\begin{gathered}
\left(\underline{\mathbf{M}}_{3}\right)^{-1} \vec{Q}_{3} \equiv\left(\underline{\mathbf{M}}_{3}\right)^{-1}\left(\begin{array}{c}
Q_{o} \\
Q_{1} \\
Q_{2}
\end{array}\right)=\left(\underline{\mathbf{M}}_{3}\right)^{-1}\left(\begin{array}{c}
+226,767 \\
+26,978 \\
-22,399
\end{array}\right) \equiv \\
\vec{g}_{3}=\left(\begin{array}{c}
g_{o} \\
g_{1} \\
g_{2}
\end{array}\right)=\left(\begin{array}{c}
+246,656 \\
-14,362 \\
+20,954
\end{array}\right),
\end{gathered}
$$

determining the constants for $N\left(Z_{A}\right)$ in Eq. [1.7a]. The coefficients for $R\left(Z_{A}\right)$, which sets the predicted number of daily new CoVID-19 cases, are:

$$
\vec{C}_{3}=\left(\begin{array}{ccc}
1 & 1 & 1 \\
0 & 1 & 1 \\
0 & 0 & 1
\end{array}\right) \vec{g}_{3}=\left(\begin{array}{c}
c_{0} \\
c_{1} \\
c_{2}
\end{array}\right)=\left(\begin{array}{c}
+253,248 \\
+6,592 \\
+20,954
\end{array}\right)
$$

determining the constants needed for $R\left(Z_{A}\right)$ in Eq. [1.7b]. Using these $\left\{g_{0}, g_{1}, g_{2}\right\}$ values along with Eq. [1.8] gives:

$$
N\left(Z_{A} \rightarrow 0\right) \equiv N[t \rightarrow \infty]=c_{0} \equiv\{253,248\}
$$

as a new predicted total number of CoVID-19 cases at the pandemic end for the EOF model, which is a $\sim 4.17 \%$ or 10,139 increase in the number of cases, compared to the EIM value of Eq. [7.1c].

Using Eq. $[6.1 \mathrm{~b}]$ for $Z_{A}[t]$, and substituting the Eq. [7.3] $\vec{C}_{3}$ values into Eq. [1.7b] gives $R\left(Z_{A}\right)$. The $\rho[t]$ in Eq. [6.1a] is derived from $R\left(Z_{A}\right)$ using Eq. $[1.7 \mathrm{~b}]$, with the resulting EOF $\rho[t]$ plotted in Figure 5, along with the $t>t_{I}$ raw data for the daily new CoVID-19 cases.

The Figure $5 E O F$ model also gives a $t<t_{I}$ extrapolation, which shows what the combination of processes would look like, if they all had been operating continuously from the CoVID-19 pandemic start. The companion $N[t]$ analytic 
result, along with the $t>t_{I}$ raw data for the total number of CoVID-19 cases is show in Figure 6.

Comparing the size and timing of the $\rho[t]$ pandemic peak, and its Day 200 value, between the EIM (Figs. 3-4) and EOF model (Figs. 5-6), gives:

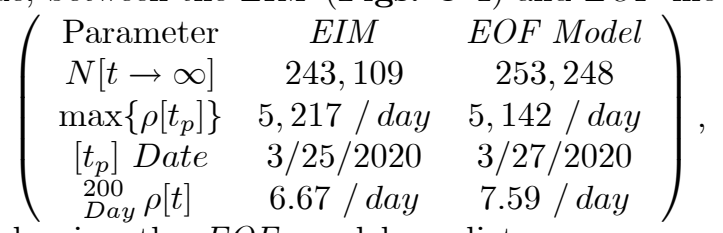

showing the EOF model predicts more cases total and more daily new CoVID-19 cases at Day 200, as well as modifying the pandemic peak predictions.

While the above analysis used $M_{F}=2$, with the Eq. [7.4] $\vec{g}_{3}$ setting the best $\left\{g_{0}, g_{1}, g_{2}\right\}$ values, this EOF model also provides estimates for the simpler $M_{F}=\{0,1\}$ cases, as outlined by Eqs. [6.14a]-[6.14f]. For $M_{F}=1$, the best two $\left\{g_{0}^{\prime}, g_{1}^{\prime}\right\}$ values were gotten by only using $\left\{Q_{0}, Q_{1}\right\}$ and an $\underline{\mathbf{M}}_{2}$ sub-matrix of $\underline{\mathbf{M}}_{3}$. For $M_{F}=0$, the best $\left\{g_{0}^{+}\right\}$by itself is derived by using $\left\{Q_{0}\right\}$ and the $\underline{\mathbf{M}}_{1}$ sub-matrix. These alternative estimates give:

$$
\begin{aligned}
& \vec{g}_{2}=\left(\begin{array}{c}
g_{0}^{\prime} \\
g_{1}^{\prime}
\end{array}\right)=\left(\begin{array}{ll}
.93432 & .10895 \\
.10895 & .51376
\end{array}\right)^{-1}\left(\begin{array}{c}
226,767 \\
26,978
\end{array}\right)=\left(\begin{array}{c}
242,584 \\
1,069
\end{array}\right) \\
& \vec{g}_{1}=\left(g_{0}^{+}\right)=(0.93432)^{-1}(+226,767)=(242,709) \text {. [7.8a]-[7.8b] }
\end{aligned}
$$

These additional calculations give the following progression of estimates for $N[t \rightarrow \infty]$, which is the final number of CoVID-19 cases at the pandemic end:

$$
\left(\begin{array}{c}
N_{A}[t \rightarrow \infty ; E I M] \\
g_{o}^{+} \\
c_{o}^{\prime}=g_{0}^{\prime}+g_{1}^{\prime} \\
c_{o}=g_{0}+g_{1}+g_{2}
\end{array}\right)=\left(\begin{array}{c}
243,109 \\
242,709 \\
243,653 \\
253,248
\end{array}\right),
$$

based on increasing the number of data fitting parameters used with the original data. This summary shows the $N[t \rightarrow \infty]$ projections are fairly stable, with an average and $1 \sigma$ standard deviation:

$$
<N[t \rightarrow \infty]>=245,680 \pm 5,060
$$

among these different calculations, where $1 \sigma$ is $\sim 2.06 \%$ of the overall average.

Comparing the results among Figs. 1-6 also highlights these items:

(a) All $\rho[t]$ functions have a sharp rise, and slower decreasing tail. The fastest changing $\rho_{\text {data }}[t]$ tail, as in the Italy CoVID-19 (Fast Shutoff) data, was successfully modeled by adding in an exponential term, as in Eq. [6.1b].

(b) The datafits in Fig. 4 and Fig. 6 show that the extra parameters in the $E I M$ and $E O F$ model fits the $\rho_{\text {data }}[t]$ shape progressively better.

(c) The EOF model shows only relatively small changes of $\sim 2.06 \%$ in the $N[t \rightarrow \infty]$ limits (Eq. [7.10]), as an estimate of uncertainty in the EIM.

(d) The Enhanced Initial Model (EIM) function captures much of the progression to a pandemic Fast Shutoff, as seen in the Italy data.

The $\rho[t]$ tail may still differ from these predictions, due to factors such as:

(i) The CoVID-19 dynamics may change in the long-term low $\rho[t]$ regime;

(ii) A "second wave" or multiple waves of $\rho[t]$ resurgence may occur, which are beyond the scope of this CoVID-19 pandemic modeling. 


\section{Summary and Conclusions}

The early stages of the CoVID-19 coronavirus pandemic starts off with a nearly exponential rise in the number of infections with time. Defining $N[t]$ as the expected total number of CoVID-19 cases vs time, this basic function:

$$
\begin{aligned}
& N_{o}[t]=1 \exp \left[+K_{A} t /\left(1+\gamma_{o} t\right)\right]=\exp \left[+G_{o}\right] \exp \left[-Z_{o}\right] \\
& Z_{o}[t] \equiv+\left[G_{o} /\left(1+\gamma_{o} t\right)\right], G_{o} \equiv\left[K_{A} / \gamma_{o}\right] \\
& \rho_{o}[t] \equiv d N_{o}[t] / d t
\end{aligned}
$$

models Social Distancing effects as a gradual lengthening of the pandemic growth doubling time, which enables pandemic shutoff with only a small population of infected persons. The Eq. [8.1b] $Z_{o}[t]$ was our Initial Model ${ }^{\mathbf{1}}$, and gives a CoVID-19 Slow Shutoff with a long-term $\rho_{o}[t]^{\sim}\left[1 / t^{2}\right]$ tail. Previously we showed ${ }^{\mathbf{1 - 2}}$ that this $Z_{o}[t]$ model fits many $N_{\text {data }}[t]$ and $\rho_{\text {data }}[t]$ cases.

However, some data had a CoVID-19 Fast Shutoff, with a $\rho_{\text {data }}[t] \sim\left[\exp \left(-\delta_{o} t\right)\right]$ exponential tail, such as in Italy ${ }^{9}$, where a Gaussian tail $\rho[t] \sim\left[\exp \left(-q_{o} t^{2}\right)\right]$ would have decreased too quickly. An Enhanced Initial Model (EIM) was developed here, using this $Z_{A}[t]$ function:

$$
\begin{aligned}
& N_{A}[t] \approx \mathbf{1} \exp \left[+G_{o}\right] \exp \left(-Z_{A}[t]\right), \\
& Z_{A}[t] \equiv+\left[G_{o} /\left(1+\gamma_{o} t\right)\right] \exp \left(-\delta_{o} t\right), \\
& G_{o} \equiv\left[K_{A} / \gamma_{o}\right], \\
& \rho_{A}[t] \equiv d N_{A}[t] / d t .
\end{aligned}
$$

We also examined if the $\exp \left(-\delta_{o} t\right)$ exponential decay could also be subject to a Slow Shutoff, giving $\exp \left[-\delta_{o} t /\left(1+\gamma_{o} t\right)\right]$ instead of $\exp \left(-\delta_{o} t\right)$, but that did not match the Italy data. To allow more data fitting parameters beyond just $\left\{K_{A}, \gamma_{o}, \delta_{o}\right\}$, an orthogonal function method was developed ${ }^{2}$ :

$$
\begin{aligned}
N(Z) & =\sum_{\substack{m=0 \\
m=M_{F}}}^{m} g_{m} L_{m}(Z) \exp [-Z], \\
R(Z) & =\sum_{m=0}^{m=M_{F}} c_{m} L_{m}(Z) \exp [-Z], \\
N(Z) & \equiv \int_{Z^{\prime}=+\infty} \int_{Z^{\prime}=Z} R^{\prime}\left(Z^{\prime}\right) d Z^{\prime}, \\
c_{M_{F}-k} & =\sum_{m=0}^{m=k} g_{m},
\end{aligned}
$$

which is applicable to a generic $Z[t]$ function, with $N[t]=N(Z[t])$, where $Z[t] \rightarrow Z_{o}[t]$ and $Z[t] \rightarrow Z_{A}[t]$ are special cases. Larger $M_{F}$ with more $\left\{L_{m}(Z) ; m=\left(0,+M_{F}\right)\right\}$ terms can match almost any arbitrary function, enabling fits to a variety of $N[t]$ and $\rho[t]$ shapes. The $\left\{g_{m} ; m=\left(0,+M_{F}\right)\right\}$ are constants determined from each dataset. The $L_{m}(Z)$ are the Laguerre Polynomials, with several important properties given in Eqs. [6.4a]-[6.5e].

Using $Z_{A}[t]$ in Eqs. [8.3a]-[8.3d] results in this Enhanced Orthogonal Function [EOF] model, which is applicable to both Slow or Fast Shutoff CoVID-19 pandemic data. The $\rho[t]$ expected number of daily new CoVID-19 cases is:

$$
N[t] \equiv \int_{t^{\prime}=\left(-1 / \gamma_{o}\right)}^{t^{\prime}=t} \rho\left[t^{\prime}\right] d t^{\prime}
$$


$\rho[t]=R\left(Z_{A}[t]\right) \frac{d Z_{A}}{d t}=R\left(Z_{A}[t]\right)\left[\frac{G_{o}}{\left(1+\gamma_{o} t\right)}\right]\left\{\delta_{o}+\frac{\gamma_{o}}{\left(1+\gamma_{o} t\right)}\right\}\left[\exp \left(-\delta_{o} t\right)\right] . \quad[8.4 \mathrm{~b}]$

Methods were developed to derive the $\left\{K_{A}, \gamma_{o}, \delta_{o}\right\}$ values, and to determine the $\left\{g_{m} ; m=\left(0,+M_{F}\right)\right\}$ and $\left\{c_{m} ; m=\left(0,+M_{F}\right)\right\}$ constants from data. Whereas our Initial Model and EIM were $M_{F}=0$ cases, the $M_{F}=2$ case was used here to examine the Italy CoVID-19 data, as an EOF model example.

The bing.com data for Italy up to $\sim 6 / 15 / 2020$ was then analyzed, with Figures 3-6 giving the new Italy results. Both the EIM and the EOF model provided good datafits, giving similar $N[t \rightarrow \infty]$ results for the final number of CoVID-19 pandemic cases, differing by only $\sim 2 \%$ at the $1 \sigma$ level.

The $\rho[t]$ post-peak behavior best indicates if a $\delta_{o} \neq 0$ model (CoVID-19 pandemic Fast Shutoff) is applicable. The $\delta_{o} \neq 0$ case likely is a second Social Distancing process, that operates along with, but is independent of the gradual pandemic doubling time changes. That doubling time change gives rise to a CoVID-19 pandemic Slow Shutoff $\left(\gamma_{o} \neq 0\right)$, and that process still operates concurrently with the $\delta_{o} \neq 0$ CoVID-19 pandemic Fast Shutoff.

This analysis shows a wide variety of CoVID-19 data can be modeled using $\left\{K_{A}, \gamma_{o}, \delta_{o}, t_{o f f s e t}\right\}$ as parameters, covering: (I) an exponential rise at CoVID19 pandemic start; (II) a gradual lengthening of doubling times for a pandemic Slow Shutoff; and (III) an exponential decay for pandemic Fast Shutoffs.

\section{$9 \quad$ List of Figures}

Figure 1: Updated Initial Model for ITALY, CoVID-19 data to 6/15/2020. Number of daily CoVID-19 cases calculated as if Social Distancing started at $3 / 2 / 2020$, but only data from $3 / 10 / 20$ actual Social Distancing start, with $N=$ 10,149 cases, was used in calculations.

Figure 2: Comparison of Initial Model Best-Fit to Measured Data. Best-fit done on Logarithmic Y-axis, using data from 3/10/20 actual Social Distancing start with $N=10,149$ cases, through $6 / 15 / 20$ with $N=237,290$.

Figure 3: Enhanced Initial Model (EIM) for ITALY CoVID-19 data to 6/15/20. EIM best fit with $N_{A}[t] \sim \exp \left(-Z_{A}[t]\right)$ using enhanced $Z_{A}[t]$ function having an exponential decay. Adding in exponential decay term gives significantly improved fit, compared to prior $O F M$.

Figure 4: Enhanced Initial Model (EIM) datafit for ITALY CoVID-19 data to 6/15/20. EIM best fit with $N_{A}[t] \sim \exp \left(-Z_{A}[t]\right)$ using enhanced $Z_{A}[t]$ function having an exponential decay. Datafit minimizing $r m s$ error on Logarithmic $Y$-axis gives significant improvement vs $O F M$.

Figure 5: Enhanced Orthogonal Functions (EOF) for ITALY CoVID-19 data to $6 / 15 / 20$. Orthogonal functions give additional parameters for further datafit improvement, using a 3 -term series $\left(M_{F}=2\right)$. Result shows EIM, by itself, provides most of the improvement.

Figure 6: Enhanced Orthogonal Functions (EOF) for ITALY CoVID-19 data to $6 / 15 / 20$. Data for total number of CoVID-19 cases versus time, compared with 3-term EOF model for $N\left(Z_{A}[t]\right)$ shows excellent match after Social Distancing start at $N=10,149$. 


\section{References}

1. https://www.MedRxiv.org/content/10.1101/2020.05.04.20091207v1, https://doi.org/10.1101/2020.05.04.20091207, "Initial Model for the Impact of Social Distancing on CoVID-19 Spread", Genghmun Eng.

2. https://www.MedRxiv.org/content/10.1101/2020.06.30.20143149v1, https://doi.org/10.1101/2020.06.30.20143149, "Orthogonal Functions for Evaluating Social Distancing Impact on Covid-19 Spread", Genghmun Eng

3. https://www.medrxiv.org/content/10.1101/2020.03.27.20043752v1, "Forecasting COVID-19 impact on hospital bed-days, ICU-days, ventilator-days and deaths by US state in the next 4 months", IHME COVID-19 Health Service Utilization Forecasting Team.

4. https://www.geekwire.com/2020/ univ-washington-epidemiologists-predict-80000-covid-19-deaths-u-s-july/ "Univ. of Washington researchers predict 80,000 COVID-19 deaths in U.S. by July", Alan Boyle, GeekWire, March 26, 2020.

5. https://www.yahoo.com/finance/news/coronavirus-modelers-raiseprojected-u-041641553.html, "Coronavirus modelers raise projected U.S. death toll and lengthen state-by-state recovery timeline", Alan Boyle, GeekWire, April 27, 2020.

6. https://covid19.healthdata.org, update of 29 April 2020.

7. http://www.healthdata.org/covid/updates

"COVID-19: What's New for May 4, 2020: Updated IHME COVID-19 projections: Predicting the Next Phase of the Epidemic", IHME COVID-19 Health Service Utilization Forecasting Team.

8. https://finance.yahoo.com/news/ pandemic-projection-puts-u-death-220824741.html, "New pandemic projection puts U.S. death toll at nearly 135,000, due to less social distancing", Alan Boyle, GeekWire, May 4, 2020.

9. www.bing.com/covid: 'Bing COVID-19 Tracker', and https://www.bing.com/covid?form=CPVD07.

10. G. N. Watson, "A Note of the Polynomials of Hermite and Laguerre", Journal of the London Mathematical Society, 13(1938), pp. 29-32.

11. J. Gillis and G. Weiss, "Products of Laguerre Polynomials", Math. Comput., 14(69), Jan. 1960, pp. 60-63

12. For correct results, calculations done by swp55.exe requires no commas in the integer matrix or vector entries, when using: ScientificWorkplace ${ }^{T M}$ Compute_EvaluateNumerically. It is due to swp55.exe also accepting European notation where 10,149 $\Leftrightarrow 10.149$. 


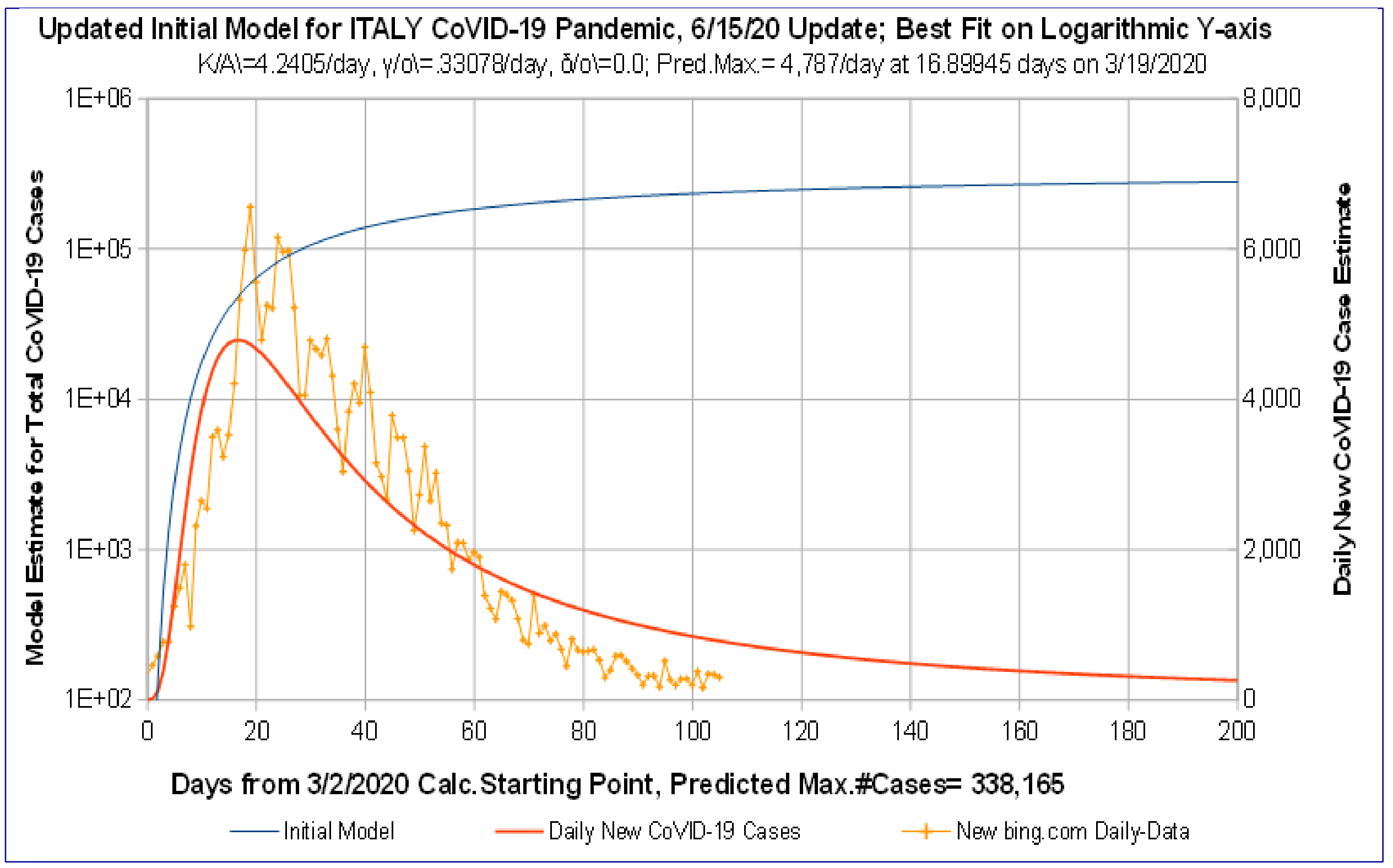

Figure 1: Updated Initial Model for ITALY, CoVID-19 data to 6/15/2020. Number of daily CoVID-19 cases calculated as if Social Distancing started at 3/2/2020, but only data from 3/10/20 actual Social Distancing start, with $N=10,149$ cases, was used in calculations. 


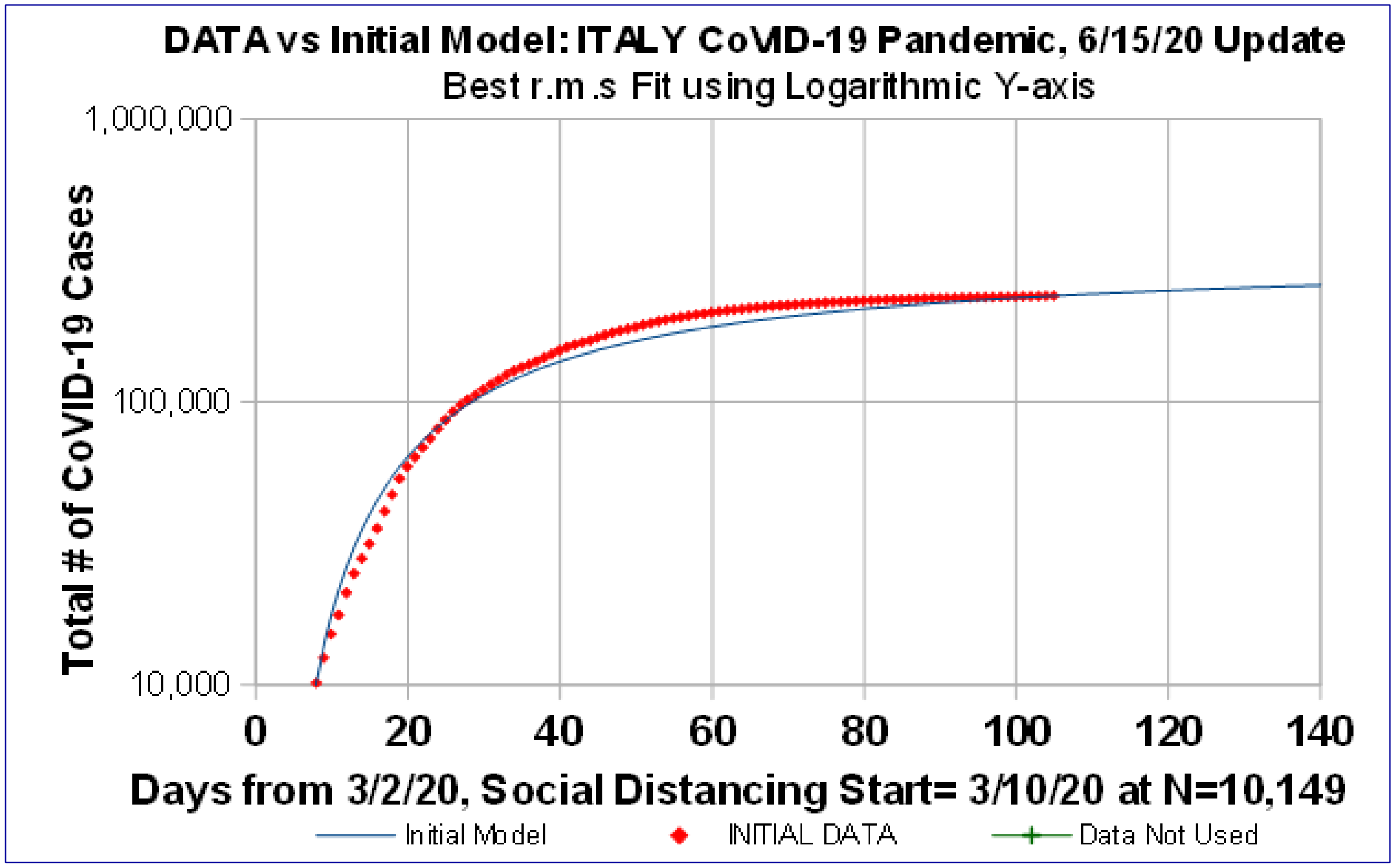

Figure 2: Comparison of Initial Model Best-Fit to Measured Data. Best-fit done on Logarithmic Y-axis, using data from 3/10/20 actual Social Distancing start with $N=10,149$ cases, through 6/15/20 with $N=237,290$. 


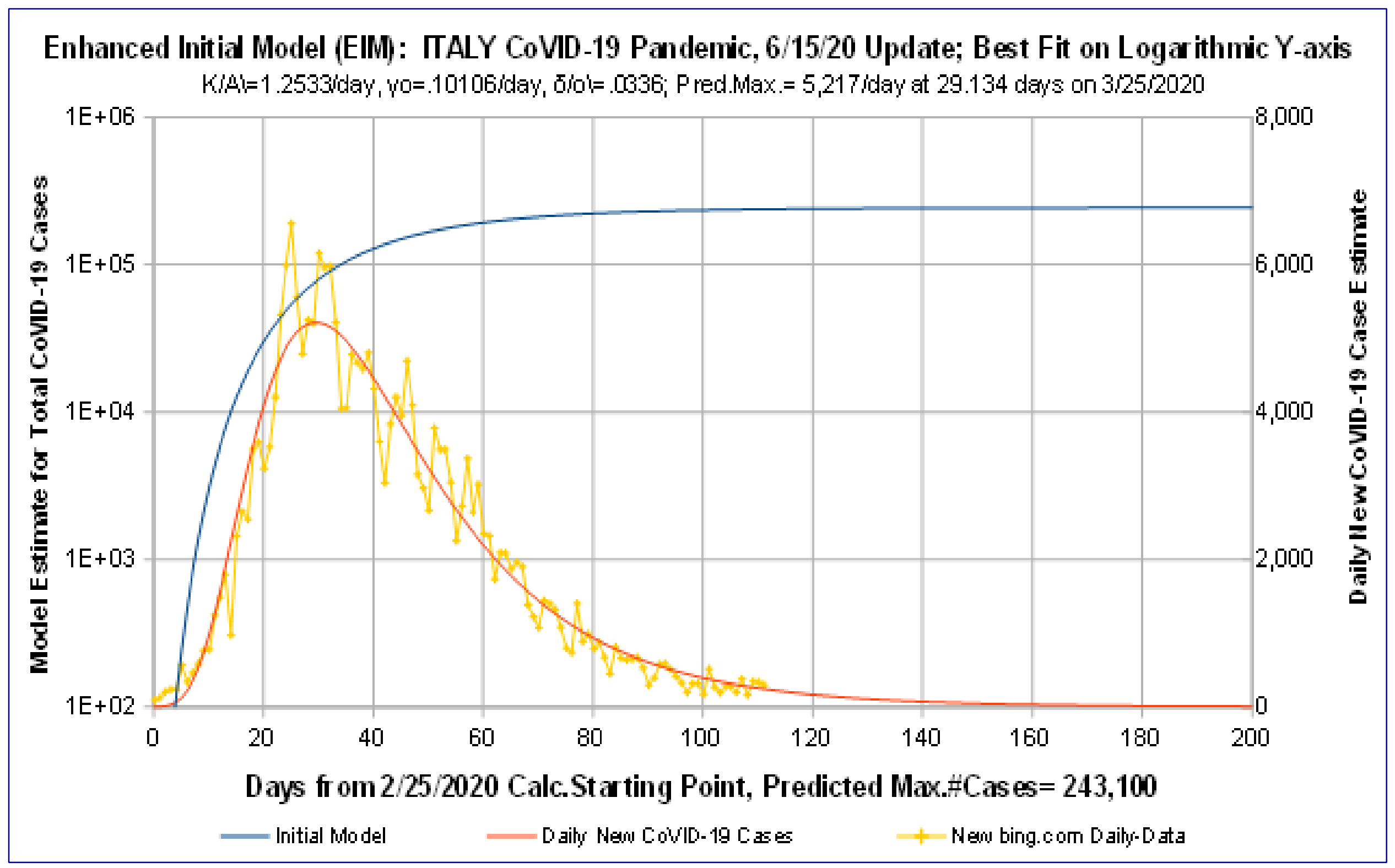

Figure 3: Enhanced Initial Model (EIM) for ITALY CoVID-19 data to 6/15/20. EIM best fit with $N_{A}[t] \sim \exp \left(-Z_{A}[t]\right)$ using enhanced $Z_{A}[t]$ function having an exponential decay. Adding in exponential decay term gives significantly improved fit, compared to prior OFM. 


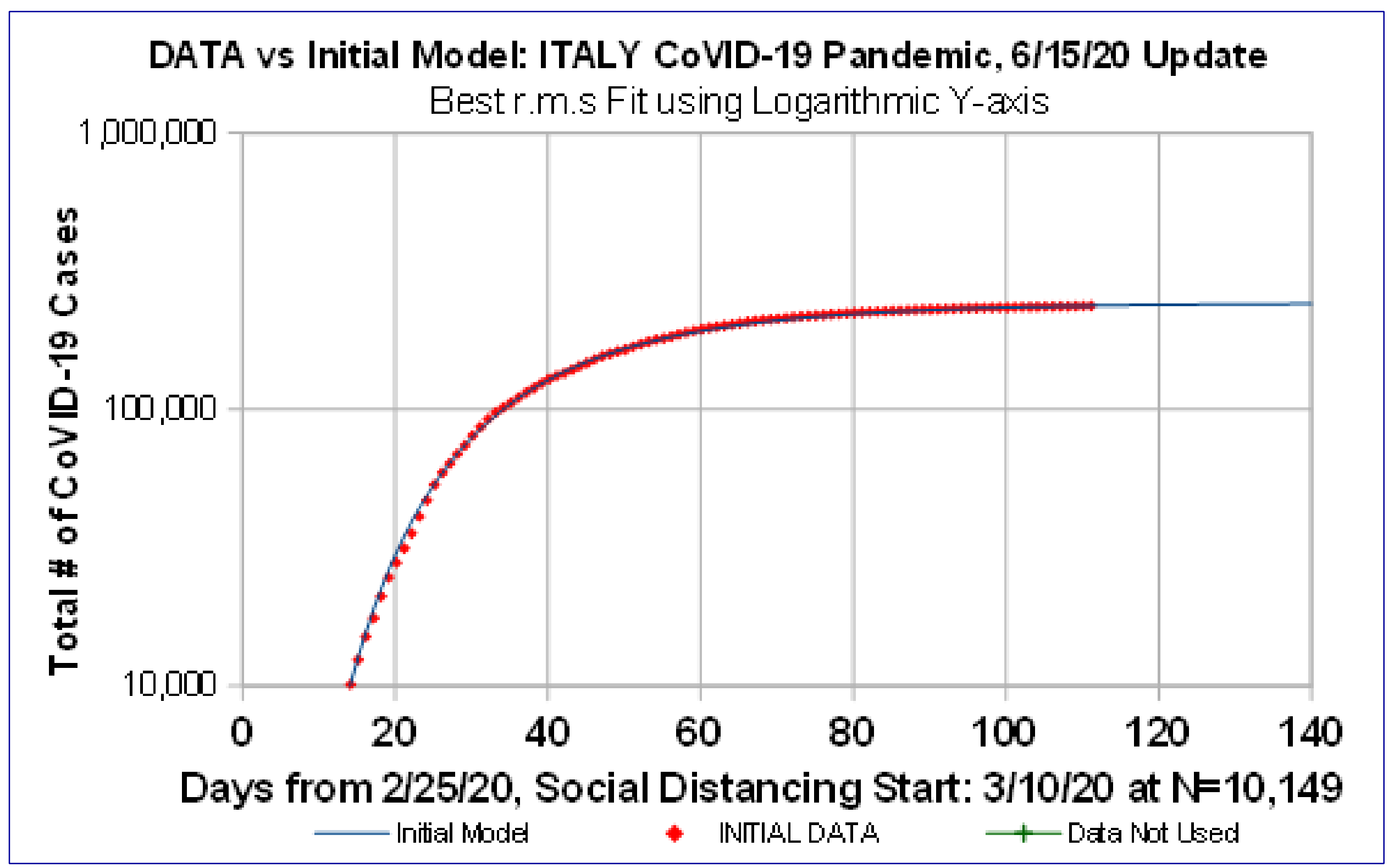

Figure 4: Enhanced Initial Model (EIM) datafit for ITALY CoVID-19 data to 6/15/20. EIM best fit with $N_{A}[t] \sim \exp \left(-Z_{A}[t]\right)$ using enhanced $Z_{A}[t]$ function having an exponential decay. Datafit minimizing rms error on Logarithmic Y-axis gives significant improvement vs OFM. 
Enhanced Orthog.Func. [E OF] Model: TTALY CoVID -19 Pandemic, 6/15/20 U pdate: Calc.Peak= 3/27/2020

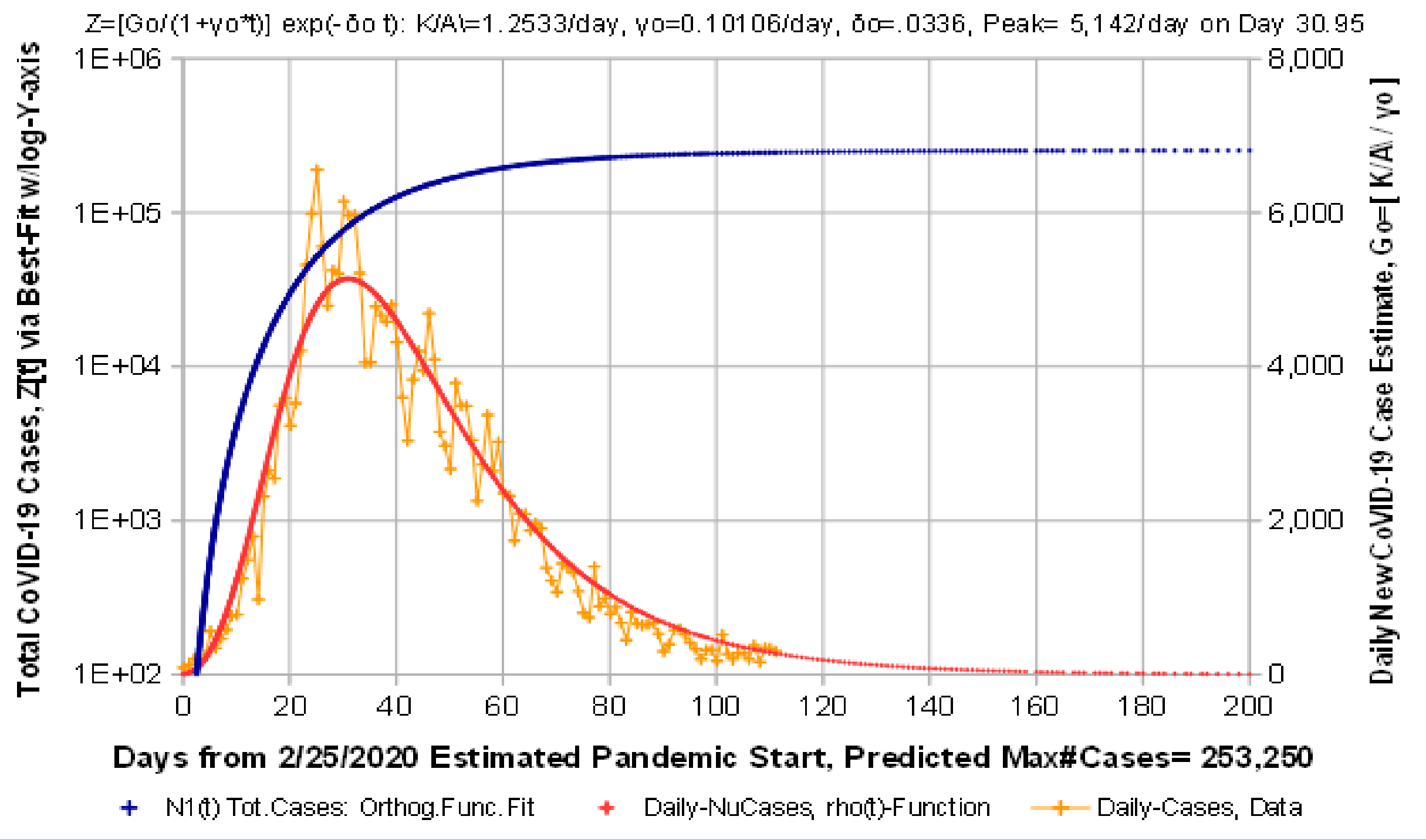

Figure 5: Enhanced Orthogonal Functions (EOF) for ITALY CoVID-19 data to 6/15/20. Orthogonal functions give additional parameters for datafit improvement, using a 3-term series $\left(M_{F}=2\right)$. Result shows $E I M$, by itself, provides most of the improvement. 


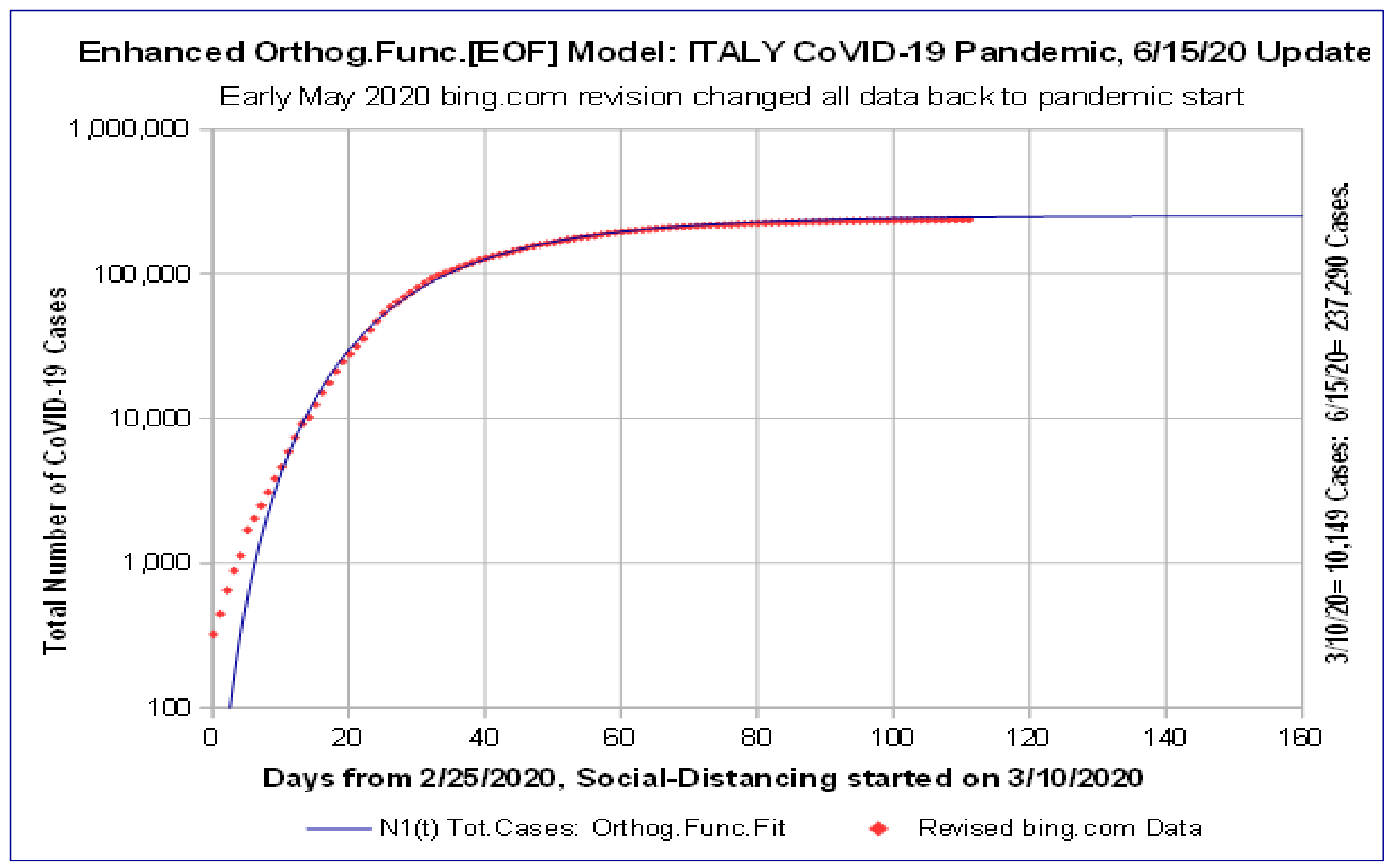

Figure 6: Enhanced Orthogonal Functions (EOF) for ITALY CoVID-19 data to 6/15/20. Data for total number of CoVID-19 cases versus time, compared with 3-term EOF model for $N\left(Z_{A}[t]\right)$ shows excellent match after Social Distancing start at $N=10,149$. 